\title{
GPPS-BJ-2019-126
}

\section{CFD MODELING OF A REALISTIC TURBOFAN BLADE FOR NOISE PREDICTION. PART 1: AERODYNAMICS}

\author{
Carlos Pérez Arroyo, Pavel Kholodov, Marlène Sanjosé, Stéphane Moreau \\ Université de Sherbrooke \\ pavel.kholodov@usherbrooke.ca \\ Sherbrooke, Quebec, Canada
}

\section{Abstract}

Various aspects of the numerical modeling of a scale-model turbofan blade are investigated for the purpose of future noise assessment. The fan geometry considered is the baseline configuration of the "Fan Noise Source Diagnostic Test" (SDT) experimental set-up. Both Reynolds Averaged Navier-Stokes (RANS) simulations and Large Eddy Simulations (LES) are performed for a single rotor blade without the stator vanes and compared against the full configuration from previous works. With RANS simulations the mesh convergence is systematically studied. In the LES, two wall models and two numerical schemes are considered to evaluate the sensitivity of the boundary-layer transition to turbulence on the blade suction side. The different LES results are compared with the RANS solutions obtained using fully turbulent and transitional turbulence models. All RANS results show similar performance, whereas in the LES the no-slip wall condition gives better performance than the log-law slip wall condition. The prism layers on the hub and the shroud change the boundary layer profile upstream of the blade but do not affect the performance. On the blade, the RANS simulations show a laminar recirculation bubble whereas the LES exhibit a leading-edge vortex spiralling radially. The transitional RANS turbulence model gives the best agreement with the LES on the pressure side. The LES results are more affected by the mesh resolution than by the wall model or the numerical scheme, especially in the tip. In the wake all results show a good agreement with the experiment.

\section{Nomenclature}

$\begin{array}{ll}\text { Variables } & \\ y^{+} & \text {Dimensionless wall distance } \\ \tau_{w} & \text { Wall shear stress } \\ C_{p}=\frac{P-P_{\infty}}{0.5 \rho_{\infty} U_{\infty}^{2}} & \text { Mean pressure coefficient } \\ C_{f}=\frac{\tau_{w}}{0.5 \rho U_{\infty}} & \text { Mean skin friction coefficient } \\ U & \text { Mean velocity } \\ P & \text { Mean pressure }\end{array}$

Indices

$$
\begin{array}{ll}
w & \text { Wall quantity } \\
\infty & \text { Free-flow quantity } \\
t & \text { Total (stagnation) quantity }
\end{array}
$$

\section{Introduction}

In high bypass ratio turbofan engine designs, the fan secondary stream has become a major contributor to the overall engine noise. The "Fan Noise Source Diagnostic Test" (SDT) experimental set-up was investigated by NASA to provide an extensive database of aerodynamic sources and aeroacoustic diagnostics of the broadband fan/OGV interaction mechanism (Hughes et al., 2002; Podboy, Krupar, Helland and Hughes, 2002; Woodward et al., 2002).

The present study is the continuation of previous works (Leonard et al., 2016; Pérez Arroyo et al., 2019, 2018), and 
gives a computational analysis with Reynolds Averaged NavierStokes (RANS) and Large Eddy Simulations (LES) for the SDT case at approach conditions for which the rotor speed is reduced and the aerodynamic characteristics are far from the design point for which the blade geometries have been optimized. This numerical study aims at comprehending and highlighting the sensitivity of the flow around the rotor blades to different numerical parameters. Two computational methods (RANS and LES), two computational domains (with, or without the rotor vanes and external flow), several mesh refinements for the RANS simulations, three turbulence models for the RANS simulations, two numerical schemes for the LES, and two boundary conditions at the blade walls for the LES are therefore considered.

The description of the simulations, including the two domains, the RANS and LES parameters, mesh definitions and cases of studies, is presented in the next section. Results are analyzed in the following section and conclusions are finally drawn.

\section{Numerical models}

\section{Domain configurations}

In this study, numerical results obtained with two different configurations are considered for the SDT Baseline approach conditions (Hughes, 2002) at 7808 revolutions per minute. In the experimental configuration, there are 22 fan blades and 54 outlet guide vanes (OGV). The first numerical configuration noted as RS (Leonard et al., 2016; Pérez Arroyo et al., 2019), includes the nacelle, the external flow and an angular periodicity of $2 \pi / 11$ with 2 fan blades. The modeling of the periodic domain shown in Fig. 1(a) became possible after the modification of the number of OGV from 54 to 55 and of their aspect ratio to maintain the same solidity. The second numerical configuration, noted as RO (Pérez Arroyo et al., 2018), focuses on the flow passage inside the nacelle to reduce the computational cost where only one rotor blade is modelled and no OGV are added, as it is shown in Fig. 1(b). The domain inlet is positioned two fan blade chords upstream of the fan leading edge (LE) and the outlet is located one OGV chord length downstream of the theoretical OGV trailing edge (TE).

\section{Mesh definitions}

The simulations are performed on four different hybrid (tetrahedra-prisms) meshes: one mesh corresponds to the RS configuration and three meshes with increasing refinement are used for the RO case. The mesh RS-V0 (RANS and LES) has

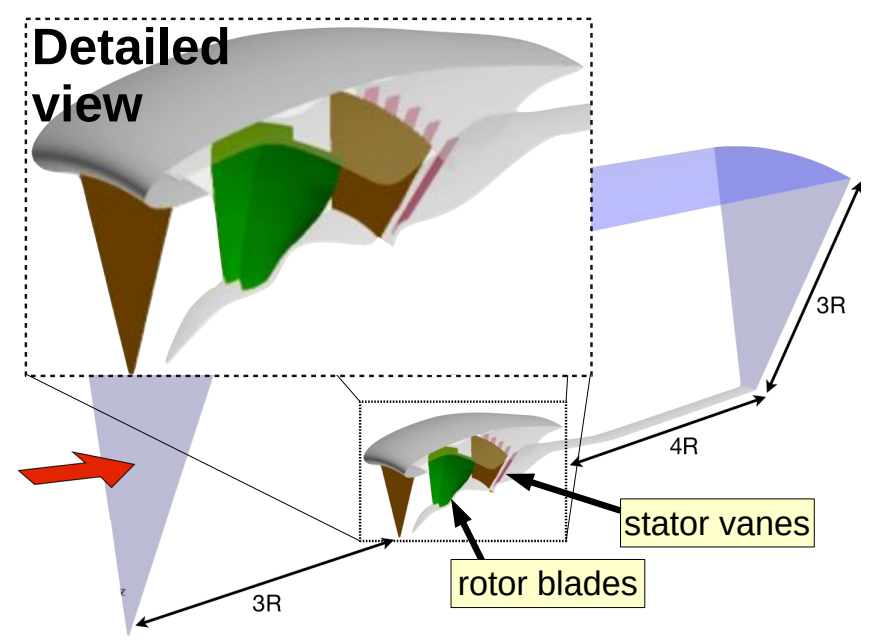

(a) Rotor-Stator configuration (RS).

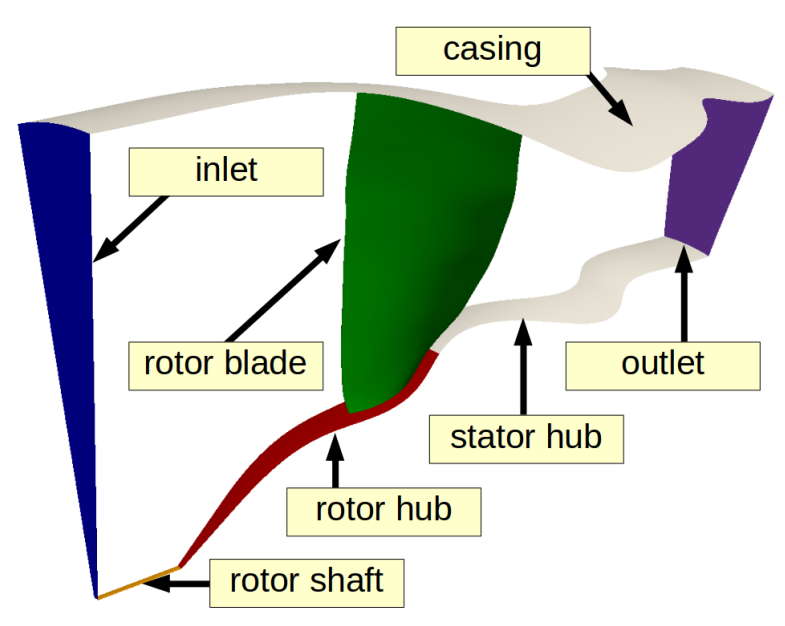

(b) Rotor-Only configuration (RO).

Figure 1 Domain views for both configurations.

a mean dimensionless distance to the wall, $y^{+}$on the blade of about 32, and tetrahedral elements on the shroud and hub. The mesh RO-V1 (RANS and LES), initially constructed for the RO LES configuration, has a similar discretization as RS-V0 close to the walls and an increased refinement upstream of the rotor and in the wake. Mesh RO-V2 (RANS) adds prism layers on the hub and shroud, and reduces the refinement upstream and in the wake. Finally, mesh RO-V3 (RANS) maintains the volume mesh parameters and increases the resolution of the laminar sub-layer having an average $y^{+} \approx 1$ on the rotor blade and $y^{+} \approx 3$ on the shroud. A detailed summary of the main mesh parameters is shown in Table 1. 


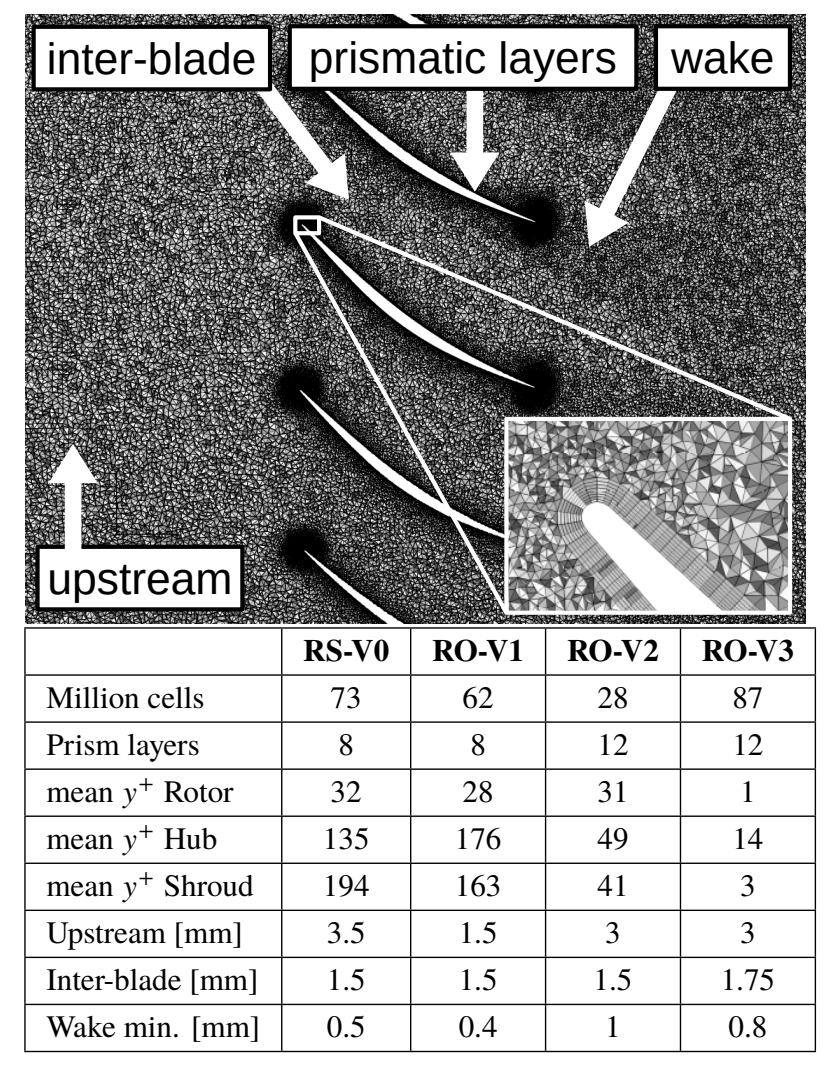

Table 1 Mesh parameters.

\section{Simulation parameters}

\section{RANS}

All the RANS simulations are performed using ANSYS CFX 17.2. The RS configuration used in (Leonard et al., 2016) with ANSYS CFX 15.0 has been recomputed for the current version to ensure the consistency between results.

For the RS RANS case, a mixing plane condition is used for the rotor-stator interface where only azimuthally averaged fluxes are exchanged (Leonard et al., 2016). For the RO cases, the total pressure, total temperature and non-dimensional components of the velocity vector extracted from the averaged RS simulation are imposed on the domain inlet. At the outlet, the mass flow-rate (scaled by the angular section) is taken from experiments. On the solid boundaries the adiabatic no slip condition is used along with the rotational speed on the rotor and hub boundaries.

For all simulations, second order accurate numerical schemes are applied. The pseudo-transient scheme is used for the steady-state computations with the pseudo time-step value of $2 \%$ of the fan rotational period. Three turbulence models are used in this work. A mesh refinement study is achieved with the standard $k-\omega$ SST model of (Menter, 1994) (mesh char- acteristics are described in the next section). In addition, the $k-\omega$ Baseline model (Menter, 1994) is also used, as it was shown to have better results for the wake width and wake turbulence on the SDT case within an unsteady RANS simulation (Shur et al., 2018). Last, LES from (Leonard et al., 2016; Pérez Arroyo et al., 2019) showed the presence of quasi-laminar flow on the pressure side of the fan blade. To check the ability of the RANS modelling to reproduce this feature, the $k-\omega$ SST Transition model (Menter et al., 2006), is tested. All above-mentioned RANS simulations also showed a too strong laminar recirculation bubble on the suction side not seen in either experiments or LES.

For all RANS computations the boundary condition on the wall is automatically scaled between the laminar wall shear stress and logarithmic wall function. The choice is based on the value of $y^{+}$. In particular, the blade and the shroud in the RO-V3 grid, having mean $y^{+}$equal to 1 and 3 correspondingly, are dominated by the laminar wall shear stress component. For other cases, the logarithmic wall function is dominating (Menter and Esch, 2001).

RANS simulations are run for 20 pseudo-rotation times. Residuals reach stable levels for the last 5 rotations with the target RMS level for each variable of $10^{-4}$. The target mass flow rate is set to be less than $0.1 \%$ of the global mass flow rate for the last 2 rotations.

\section{$\underline{\text { LES }}$}

The AVBP code developed by CERFACS and IFPEN (Schönfeld and Rudgyard, 1999) has been used for the compressible LES. The influence of two numerical schemes is investigated in this work: an explicit Lax-Wendroff (LW) $2^{\text {nd }}$ order scheme both in space and time, and a two-step TaylorGalerkin finite-element scheme named TTG4A (Quartapelle and Selmin, 1993) of $3^{\text {rd }}$ and $4^{\text {th }}$ order in space and time respectively. The sub-grid scale model used is the Wall-Adapting Local Eddy-viscosity model (WALE) that provides the proper turbulence decay toward the walls.

Two instances of solver are run simultaneously for the RS case, one for the rotor domain and one for the stator region and they are coupled by the parallel code coupler OpenPALM (Duchaine et al., 2015) which interpolates and exchanges the residuals at each iteration on overlapping grids (Wang et al., 2014). At the inlet and outlet boundary conditions of the RS configuration, Navier-Stokes characteristic non-reflective boundary conditions, combined with a sponge layer, are used to avoid spurious reflections from vortical fluctuations (Poinsot 
and Lele, 1992).

For the RO case, the inlet and outlet conditions are based on characteristics (ingoing and exiting waves) and defined with a relaxation factor towards the mean velocity and temperature at the inlet and pressure at the outlet of the RS configuration (Leonard et al., 2016).

While the rotor hub and the blade walls are rotating with the domain, the boundary condition for the stator hub defines a contra-rotating wall in the Arbitrary Lagrangian-Eulerian methodology and periodic conditions are set on the lateral sides of the domain. The boundary condition at the shaft is defined as wall slip. On the blade, two boundary conditions are used: no slip and log-law slip model (Jaegle et al., 2010). On the other walls, the log-law slip model is applied for all cases. The log-law slip model computes the wall shear stress from the log-law function and imposes non-zero tangential velocity on the wall. The noslip model imposes zero velocity at the wall and computes the wall shear stress directly. Some numerical oscillations at the blade walls that require further investigations were observed when TTG4A was used with the WALE subgrid scale model and the log-law slip model. In order to ascertain the influence of the wall law condition, results with LW and wall law from previous works (Pérez Arroyo et al., 2018) are also analyzed.

The time step of the RS and RO simulation are set to approximately $2.5 \times 10^{-5}$ and $1.2 \times 10^{-5} \mathrm{~ms}$ respectively to have a local CFL number based on the Jacobian flux tensor of the numerical scheme, below 0.7. The simulation is started from the converged LW simulation with wall law (Pérez Arroyo et al., 2018). After a transient time of 4 revolutions the statistics have been extracted from the simulation during 9 rotations for the RS case, 3.3 for the LW with wall law case, and 5 rotations for the no slip cases. Nonetheless, all statistics are well converged after 2 rotations and only minor modifications are visible.

\section{Cases of study}

In this work 10 cases, from which 6 are RANS and 4 are LES, are compared and analyzed. In terms of RANS simulations, the $k-\omega$ SST model is used to study the effect of different mesh discretization with the meshes RO-V1, RO-V2 and ROV3 with increasing refinement on the walls. The $k-\omega$ SST Transition model and $k-\omega$ Baseline model are computed only on the refined RO-V3 mesh. In addition, the RS case is also compared. The LES cases correspond to the RS case from previous works (Leonard et al., 2016; Pérez Arroyo et al., 2019) with LW and wall laws on the blades, the RO case from (Pérez Arroyo et al., 2018) with LW and wall laws and two new simulations for the RO cases with no slip conditions without wall laws on the blades, LW and TTG4A numerical schemes. All simulations are summarized in Table 2 along with the mass flow rate $\dot{m}$ and the total pressure ratio $P_{t} / P_{a m b}$ after the rotor. The RS configuration gives a $\dot{m}$-relative error with respect to experiments higher than $2 \%$ for both RANS and LES, nonetheless, the $P_{t} / P_{a m b}$ is well computed for RANS and with only $0.25 \%$ difference for the LES. On the other hand, the RO configuration in RANS, matches perfectly the mass flow rate, as it is imposed at the outlet boundary condition and underestimates the pressure ratio difference by less than $0.30 \%$. As the boundary condition is not the same for the LES (RO), the $\dot{m}$ varies about $1 \%$ and the pressure ratio is overestimated by less than $0.5 \%$ ( $0.17 \%$ for the no slip conditions).

\begin{tabular}{|l|c|c|l|}
\hline Case & Conf. & Mesh & Parameters \\
\hline RS-RANS & RS & RS-V0 & $k-\omega$ SST model \\
\hline RANS-V1-SST & RO & RO-V1 & $k-\omega$ SST model \\
\hline RANS-V2-SST & RO & RO-V2 & $k-\omega$ SST model \\
\hline RANS-V3-SST & RO & RO-V3 & $k-\omega$ SST model \\
\hline RANS-V3-Trans & RO & RO-V3 & $k-\omega$ SST Transition model \\
\hline RANS-V3-Base & RO & RO-V3 & $k-\omega$ Baseline model \\
\hline RS-LES & RS & RS-V0 & LW, wall law on blade \\
\hline LES-LW-WL & RO & RO-V1 & LW, wall law on blade \\
\hline LES-LW-NS & RO & RO-V1 & LW, no slip on blade \\
\hline LES-TTG4A-NS & RO & RO-V1 & TTG4A, no slip on blade \\
\hline
\end{tabular}

Cases description

\begin{tabular}{|l|c|c|}
\hline Case & $\dot{\boldsymbol{m}} \mathbf{( k g / s )}$ & $\boldsymbol{P}_{\boldsymbol{t}} / \boldsymbol{P}_{\boldsymbol{\infty}}$ \\
\hline RS-RANS & 25.86 & 1.159 \\
\hline RANS-V1-SST & 26.44 & 1.156 \\
\hline RANS-V2-SST & 26.44 & 1.156 \\
\hline RANS-V3-SST & 26.44 & 1.155 \\
\hline RANS-V3-Trans & 26.44 & 1.156 \\
\hline RANS-V3-Base & 26.44 & 1.157 \\
\hline RS-LES & 25.77 & 1.162 \\
\hline LES-LW-WL & 26.73 & 1.164 \\
\hline LES-LW-NS & 26.73 & 1.161 \\
\hline LES-TTG4A-NS & 26.74 & 1.161 \\
\hline Experiments & 26.44 & 1.159 \\
\hline
\end{tabular}

Performance results

Table 2 Summary of the cases.

\section{Results}

\section{Hub and shroud boundary layers}

The boundary layer profiles for the Mach number in the rotating reference frame and the turbulence kinetic energy are presented in Figs. 2 and 3. The profiles are taken as a result of an azimuthal averaging $10 \%$ of the rotor chord length upstream of the rotor LE (Fig. 2) and 10\% of the rotor chord length 
downstream of the rotor TE (Fig. 3).

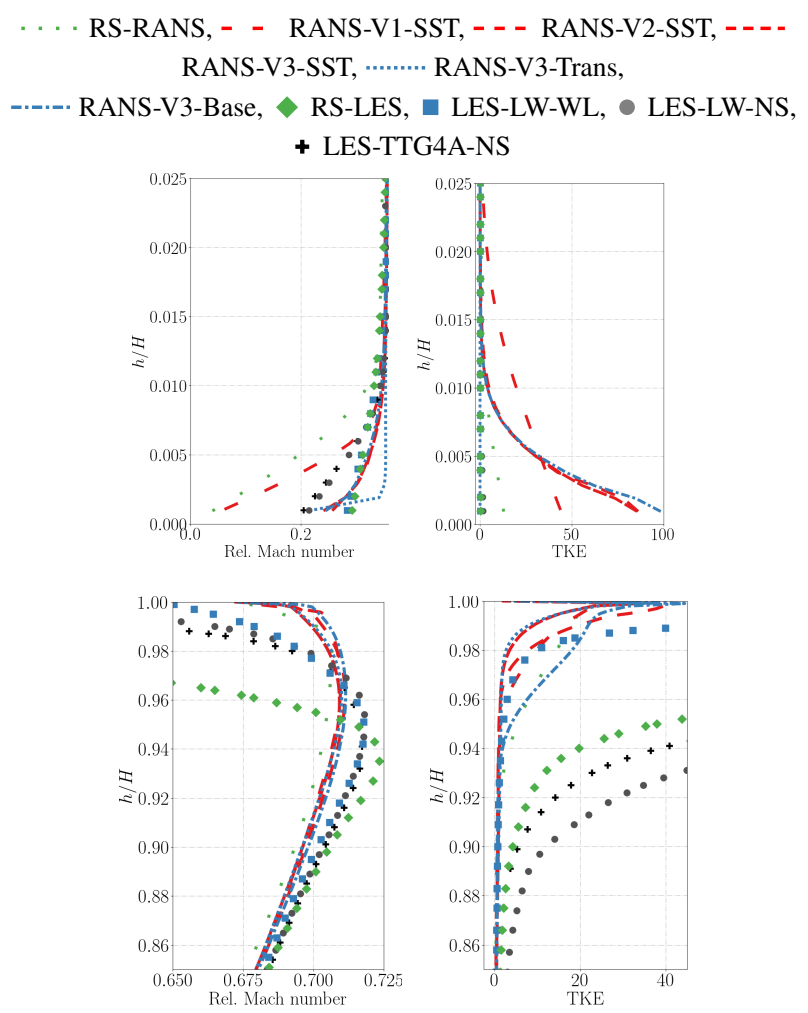

Figure 2 Azimuthally averaged profiles over the span of the relative Mach number and TKE at $10 \%$ of the rotor chord length upstream of the rotor LE.

Figure 2 (upper line) shows that the addition of the prism layers on the hub surface (RO-V2 and RO-V3) results in a thinner boundary layer on the hub for all observed values of $y^{+}$. Moreover, the transition model gives a more turbulent boundary layer than any other model. Large Eddy Simulations stay close to the RANS results with prisms and are grouped depending on whether or not they have a wall law model, with a thicker boundary layer if the condition is no slip, independently of its numerical scheme or domain configuration. The Mach number profiles away from the hub merge together for all cases, hence, the upstream mesh resolution investigated in the current research does not influence the core profile upstream of the rotor blades. The TKE near the hub depicts a similar behavior as the Mach number in terms of prism layers. Simulations with tetrahedral elements on the walls show a thicker boundary layer and lower values of TKE than the ones with prism layers and all results with the exception of RANS-V3-Trans are similar. The transition model, gives close to zero TKE levels which is in agreement with all LES simulations.

On the shroud (Fig. 2, bottom line), all RANS simulations give similar profiles that differ from all LES simulations. Moreover, the effect of the domain configuration is well depicted. The
LES on RO-V1 presents comparable results whereas the RSLES shows a different profile. At $h / H>0.86$, the boundary layer development is influenced by the geometry of the nacelle. The RO simulations inlet profiles are extracted from the averaged RS solution which removes all the unsteady flow generated by the actual nacelle inlet hence impacting the boundary layer on the shroud. The TKE near the shroud, illustrates a high sensitivity to the different parameters from the cases as mostly all results present different shapes. Only RANS-V3-SST and RANS-V3-Trans provide the same profile. On the other hand, even when keeping the same mesh, RANS-V3-Base shows profiles that are closer to RS-RANS than any other case. For LES, it can be seen as a combined effect of the wall laws, the refinement and the domain configuration. LES-LW-WL depicts similar results as the RANS. On the other hand, RS-LES, even though it uses wall laws, a coarser mesh and the different inlet conditions give a profile that is fortuitously close to the no-slip cases.

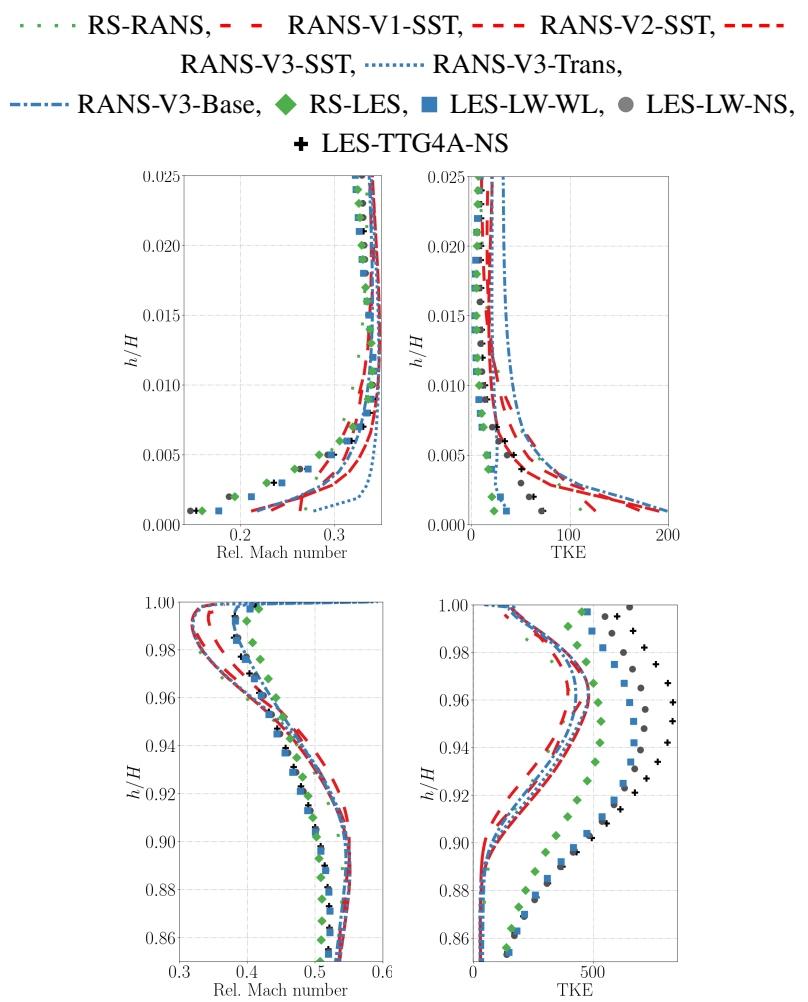

Figure 3 Azimuthally averaged profiles over the span of the relative Mach number and TKE at $10 \%$ of the rotor chord length downstream of the rotor TE.

After the rotor blade, the Mach number profiles near the hub exhibit similar results for the RANS where three distinct groups can be found in Fig. 3 (upper line). Results without prism layers show a profile almost perpendicular to the wall. RANS-V3Trans gives a thinner boundary layer and the rest of the cases 
provide similar profiles. On the other hand, all LES present the same results. The TKE, follows the same trends as upstream of the rotor, albeit showing a more marked difference between the different refinements. RANS-V3-Trans matches the LES results with wall laws which give lower levels of TKE with respect to the no-slip cases.

Near the shroud, focusing on above $h / H>0.94$ in Fig. 3 (bottom left) three different behaviors can be seen. Meshes RS-V0, RO-V1 and RO-V3 give similar results for all cases but RANS-V3-Base. On the other hand, RO-V2 is positioned between the previous two groups. This phenomena can be explained by the fact that, when the wall is refined for ROV3 it also implies that the volume mesh refinement adjacent to the rotor and shroud walls is increased in order to prevent mesh quality deterioration. Hence, the wall mesh resolution is shown not to influence the profiles downstream of the rotor blades. Yet, the latter profiles at the tip are influenced by the wake refinement. For this reason, results for RS-V0 and RO-V3 are similar. At $h / H<0.9$, all the RANS cases show similar profiles indicating that in the core region the difference in mean results due to the wake mesh resolution is negligible. LES cases depict closer profiles with a slight difference between RS-LES and the other simulations. The TKE profiles downstream of the rotor in Fig. 3 (bottom right) show similar results for all RANS cases and higher values of TKE for the LES. At $h / H>0.90$, the flow encounters the wake vortical structures developed at the rotor LE that are convected towards the shroud (see Fig. 7 in (Pérez Arroyo et al., 2018)) and the tip-gap flow. The effect of refinement and numerical scheme with a higher order is illustrated by an increase in the TKE levels.

The LES results also exhibit an increase in TKE near the wall (Fig. 3, bottom right). It is linked with the coarse near-wall resolution where the last points on the depicted curves are located far from the wall. Moreover, the wall law condition applied on the shroud in combination with the WALE subgrid model results in non-zero tangential velocity fluctuations which also affects the TKE value near the wall (Schmitt, 2005).

\section{Rotor surface flow analysis}

The mesh refinement on the blade is highlighted by flow analysis on the surface of the blade. The profiles of pressure coefficient $C_{p}$ at $50 \%$ of the span are presented in Fig. 4. Globally, all cases present the same distribution in terms of shape and levels. Only noticeable differences can be seen in the region of $x / c<0.15$ for the suction side. At $x / c \approx 0.05$, a small three-dimensional separation bubble appears which changes the $C_{p}$ profiles. For RANS simulations, meshes RS-V0, RO-
V1 and RO-V2 capture similar phenomena and a separation bubble of length $\Delta x / c=0.086$ (computed from the friction coefficient). In addition, RANS-V3-Base depicts similar values than the coarser meshes for $x / c>0.05$ but different shape for $x / c<0.05$. Once the mesh is refined up to $y^{+} \approx 1$ (RO$\mathrm{V} 3$ ), the profiles change completely, giving longer plateau in $C_{p}$ and thus a longer separation bubble of about $\Delta x / c=0.125$. In the LES, no clear plateau can be seen and the recirculation bubble rather develops as a leading-edge vortex in the spanwise direction. Nonetheless, this vortex trace has the same length. RO-LES results show no appreciable impact from the numerical scheme nor the boundary condition. On the other hand, RS-LES give values similar to those obtained with the coarser meshes in RANS.

The absolute friction coefficient $C_{f}$ on the blades is shown in Fig. 5 for selected representative cases. On the suction side, all RANS contours presents similar patterns with lower levels for the coarser mesh RO-V1. The separation bubble can be seen to develop along the full span leading to a reattached turbulent boundary layer with a peak in $C_{f}$ at about $h / H \approx 0.75$. The flow then becomes turbulent in the upper section of the blade and reaches the TE. A similar behavior near the LE is found for the LES simulations. However, the higher order of the TTG4A numerical scheme depicts several spots with increased $C_{f}$ along the LE. The maximum of $C_{f}$ is found at the same location as in the RANS but the flow is less turbulent. On the pressure side, the flow becomes turbulent on the top half of the blade for the $k-\omega$ SST model whereas the $k-\omega$ SST Transition model depicts a laminar flow with striation patterns that are also captured by the LES. The LES numerical scheme does not change significantly the $C_{f}$ patterns.

\section{Wake flow analysis}

To better understand the wake resolution impact on the mean results, the wake profiles of the axial velocity and RMS are presented in Fig. 6 at three $h / H$ positions. Results are compared with hot-wire measurements of Podboy, Krupar, Hughes and Woodward (2002) (low-noise configuration with swept OGV). The axial velocity profiles are normalized by the velocity outside the wake and centred at the wake deficit minimum for an easier comparison.

At $10 \% \mathrm{~h} / \mathrm{H}$ close to the hub, the turbulence is very weak and the results show a thin wake. The axial velocity deficit is overpredicted by all RANS simulations except RANS-V3-Trans that overpredicts it and gives results similar to those obtained with the LES. Moreover, only RANS-V1-SST correctly predicts the hump on the suction side (positive $\theta$ ). The LES show similar 


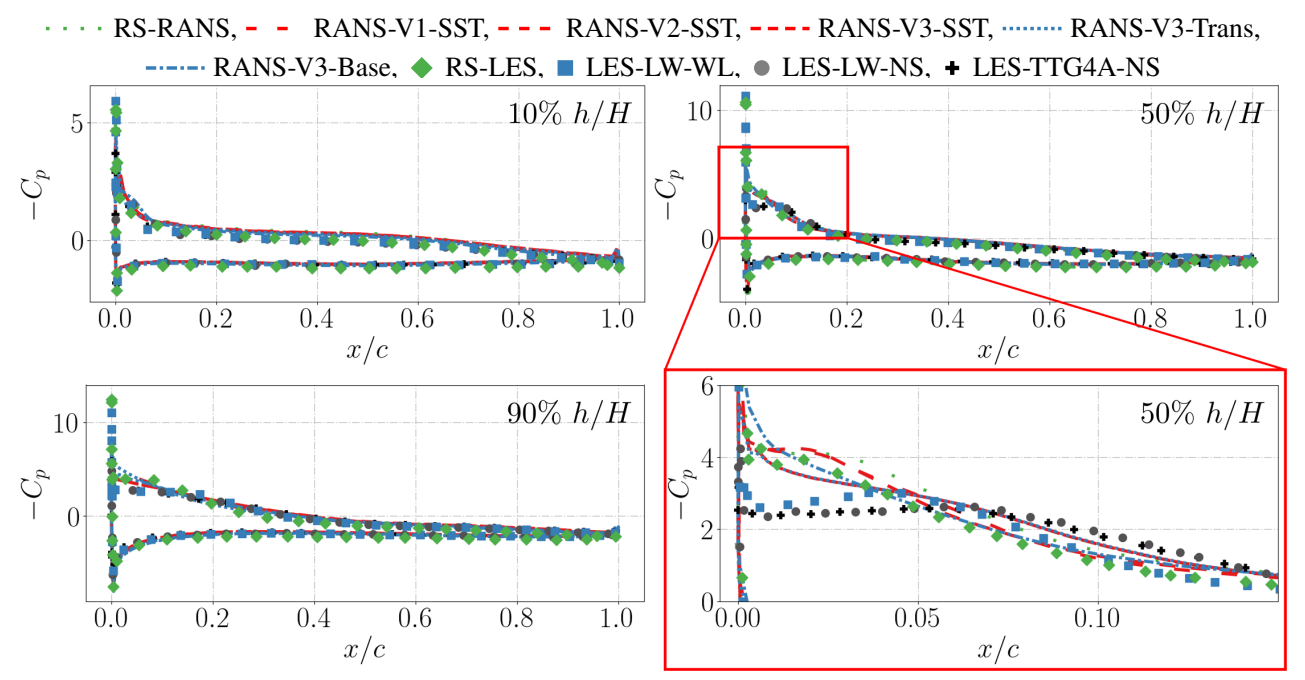

Figure 4 Pressure coefficient profiles at different location along the span computed with reference values taken at $10 \%$ chord upstream of the rotor LE.
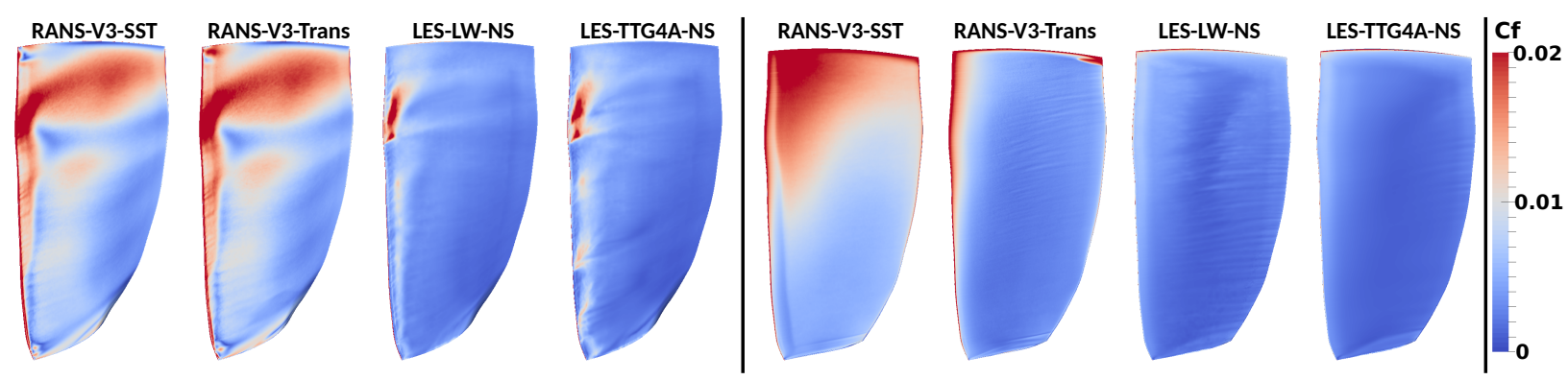

Figure 5 Absolute friction coefficient computed with reference values at $10 \%$ of the rotor chord length upstream of the rotor LE. Left: suction side. Right: pressure side. Flow goes from left (LE) to right (TE).

results with a velocity deficit underpredicted and the thinnest wake is obtained for LES-LW-WL.

For the axial RMS velocity profiles at $10 \% \mathrm{~h} / \mathrm{H}$ all RANS profiles give results close to the experiments whereas LES results, overpredict the RMS peak with the exception of LES-LW-WL which gives the correct levels. Moreover, at this location, the experimental maximum is obtained on the pressure side and not on the suction side as expected (LES-LW-NS).

At $50 \% h / H$, the axial velocity deficit of all RANS results agree well with experiments with RANS-V2-SST giving the best match. However, the wake width is underpredicted for all RANS cases. The trend observed for the RANS results is the convergence towards the slightly smaller wake width compared to the experiment. All LES underpredict the velocity deficit, but better capture the width of the wake and the RMS profile. The numerical scheme does not affect the wake, most likely due to the density of the mesh, but the use of wall law changes the slope of the velocity profile on the pressure side (negative $\theta)$.
For the axial RMS velocity profiles at $50 \% h / H$, all the RANS underpredict velocity fluctuations with results progressively approaching the experiments (thick black solid line) with increasing refinement of the wake. The RMS values are computed assuming isotropic turbulence and they show similar levels as the experimental ones computed with an analogous procedure (thin grey solid line). LES results present a better agreement with experiments than RANS with a slight overprediction in the RMS peak and a broader hump. The numerical scheme does not have an influence.

Finally, at $90 \% \mathrm{~h} / \mathrm{H}$, the flow is fully turbulent and the wake highly destabilized by the tip-gap flow. RANS results show that they are able to capture the velocity deficit but they overpredict the pressure side, and underpredict the suction side of the wake. None of the LES results are able to predict the shape of the experimental profile, and these are impacted by the mesh and the domain configuration, but not by the numerical scheme.

The average levels for the RANS simulations are in agreement with experiments. There is only minor difference between the RANS predictions in the tip region (Fig. 6,f). The minimum 


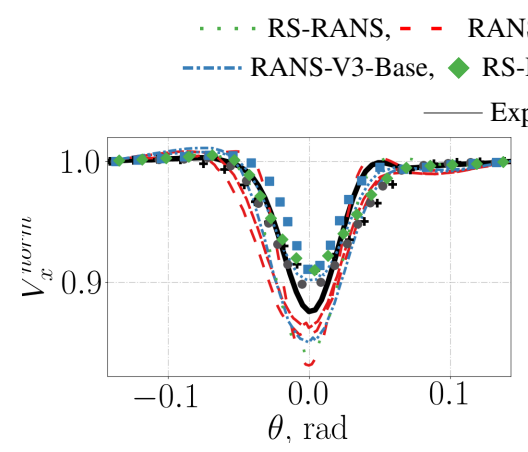

(a) $10 \% \mathrm{~h} / \mathrm{H}$

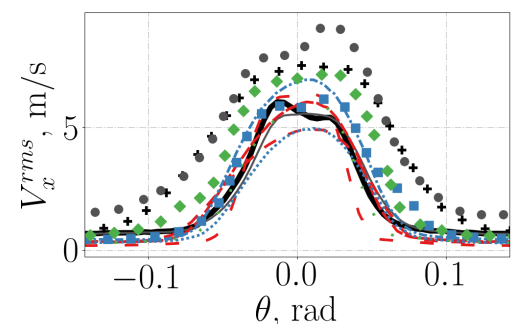

(d) $10 \% \mathrm{~h} / \mathrm{H}$

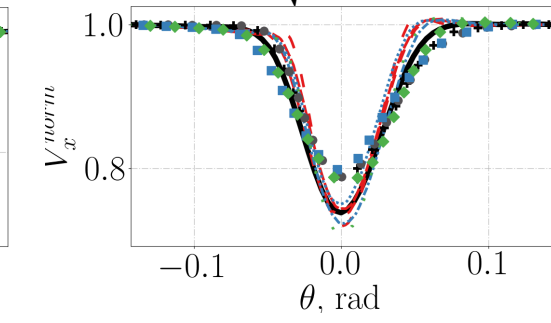

(b) $50 \% \mathrm{~h} / \mathrm{H}$

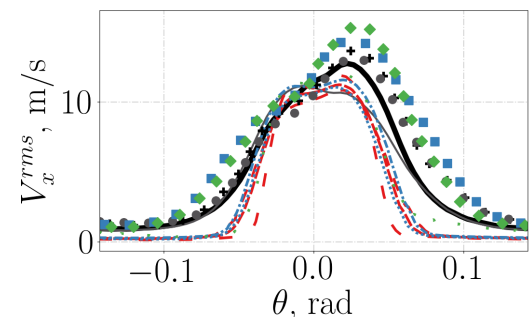

(e) $50 \% h / H$

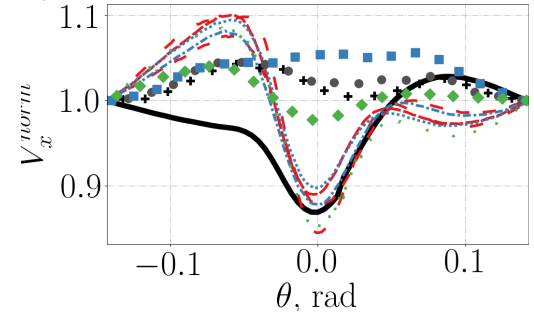

(c) $90 \% \mathrm{~h} / \mathrm{H}$

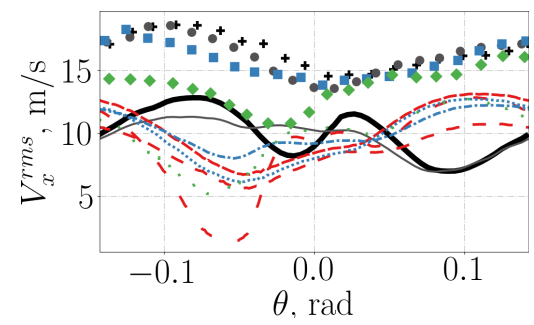

(f) $90 \% h / H$

\section{Figure 6 Wake velocity profiles at mid-distance between the rotor TE and the theoretical stator LE. Left: Axial velocity normalized by the external velocity of the wake. Right: RMS RANS velocity computed from TKE.}

of RMS values is lower for RO-V1 mesh, but elsewhere all three configurations give the same result. At the same time the profiles do not follow the experimental trends. A possible explanation is the unsteady character of the tip gap flow. The steady-state RANS simulations cannot properly resolve this unsteady phenomenon. Another reason is that the tip gap resolution is still insufficient. It is also the case of LES where the results are insensitive to the wall modeling. The LES average levels are increased by $10 \%$. All LES except RS-LES give similar results. It can be the result of slightly better wake resolution for RO meshes compared to the RS-V0 mesh.

\section{Conclusions}

The present work focused on the rotor flow topology of the "Fan Noise Source Diagnostic Test" (SDT) NASA configuration at approach conditions. A detailed study of the mesh and turbulence model has been performed with RANS simulations. These RANS results have been compared with LES simulations involving two mesh configurations, two wall models and two numerical schemes. Between the RANS simulations the performance results are very similar. The Rotor Only meshes generally give better mass flow rate prediction than in the Rotor-Stator RANS simulation due to different outlet boundary condition. There is no clear impact of the RANS model on the performance results. The LES with the no-slip wall boundary condition have better prediction than the walllaw configurations. The prism layers on the hub and shroud surfaces modify the boundary layer profiles of the flow upstream of the blade. However, it yields no difference in the performance predictions. Hence, the rotor blade modeling has the major impact on the performance.

On the blade, the RANS results indicate a laminar separation bubble which is not observed in the LES results. Instead, the latter show the sign of a spanwise vortex roll-up. The LES are influenced by the resolution on the blade, less by the the wall modeling or the numerical scheme precision. On the contrary, the RANS show minor changes with mesh resolution, while the turbulence closure influences the boundary layer flow development. The $k-\omega$ SST Transition model shows the best match with LES reproducing the quasi-laminar flow on the pressure side.

All LES show roughly similar wake deficit but overpredict the RMS values. The influence of the numerical scheme is seen only at $h / H=10 \%$ where TTG4A scheme gives better peak values with respect to the experiments. The RANS results tend to converge to slightly thinner wake width with the mesh refinement. In general, both RANS and LES agree well with experimental data. This analysis provides an exhaustive computational database for the subsequent broadband noise prediction using the RANS and LES data in the analytical models of aeroacoustics. 


\section{Acknowledgments}

The computations were made on the supercomputer Mammouth-MP2 from Université de Sherbrooke, managed by Calcul Québec, Graham from University of Waterloo managed by WestGrid and Niagara from University of Toronto managed by managed by Scinet, all parts of Compute Canada's national platform of Advanced Research Computing resources. The authors want to thank CERFACS, which develops and provides us with AVBP for academic research.

\section{References}

Duchaine, F., Jauré, S., Poitou, D., Quémerais, E., Staffelbach, G., Morel, T. and Gicquel, L. (2015), 'Analysis of high performance conjugate heat transfer with the OpenPALM coupler', Computational Science \& Discovery 8(1), 015003.

Hughes, C. (2002), Aerodynamic performance of scale-model turbofan outlet guide vanes designed for low noise, in "40th AIAA Aerospace Sciences Meeting \& Exhibit', AIAA Papers, Reno, NV, USA.

Hughes, C., Jeracki, R., Woodward, R. and Miller, C. (2002), Fan Noise Source Diagnostic Test - Rotor Alone Aerodynamic Performance Results, in '8th AIAA/CEAS Aeroacoustics Conference \& Exhibit', AIAA-2002-2426, AIAA papers, Breckenridge, CO, USA.

Jaegle, F., Cabrit, O., Mendez, S. and Poinsot, T. (2010), 'Implementation Methods of Wall Functions in Cell-vertex Numerical Solvers', Flow, Turbulence and Combustion 85(2), 245-272.

Leonard, T., Sanjosé, M., Moreau, S. and Duchaine, F. (2016), Large Eddy Simulation of a scale-model turbofan for fan noise source diagnostic, in '22nd AIAA/CEAS Aeroacoustics Conference', AIAA Paper 2016-3000, American Institute of Aeronautics and Astronautics.

Menter, F. (1994), 'Two-equation eddy-viscosity turbulence models for engineering applications', AIAA Journal 32(8), 1598-1605.

Menter, F. and Esch, T. (2001), Elements of industrial heat transfer predictions, in '16th Brazilian Congress of Mechanical Engineering'.

Menter, F., Langtry, R., Likki, S., Suzen, Y., Huang, P. and Völker, S. (2006), 'A correlation-based transition model using local variables-part I: model formulation', Journal of turbomachinery 128(3), 413-422.
Pérez Arroyo, C., Leonard, T., Sanjose, M., Moreau, S. and Duchaine, F. (2019), 'Large Eddy Simulation of a Scalemodel Turbofan for Fan Noise Source Diagnostic', Journal of Sound and Vibration 445, 64-76.

Pérez Arroyo, C., Sanjose, M., Moreau, S. and Duchaine, F. (2018), Large Eddy Simulation of a Rotor Stage for Fan Noise Source Diagnostic, Zenodo.

URL: https://doi.org/10.5281/zenodo.1345496

Podboy, G., Krupar, M., Helland, S. and Hughes, C. (2002), Steady and Unsteady Flow Field Measurements within a NASA 22 inch fan model, in '40th AIAA Aerospace Sciences Meeting \& Exhibit’, AIAA-2002-1033, AIAA papers, Reno, NV, USA.

Podboy, G., Krupar, M., Hughes, C. and Woodward, R. (2002), Fan Noise Source Diagnostic Test - LDV Measured Flow Field Results, in '8th AIAA/CEAS Aeroacoustics Conference \& Exhibit', AIAA-2002-2431, AIAA Paper, Breckenridge, $\mathrm{CO}$, USA.

Poinsot, T. and Lele, S. (1992), 'Boundary conditions for direct simulations of compressible viscous flows', Journal of Computational Physics 101(1), 104-129.

Quartapelle, L. and Selmin, V. (1993), 'High-order TaylorGalerkin methods for nonlinear multidimensional problems', Finite Elements in Fluids 76(90), 46.

Schmitt, P. (2005), Thermally accurate LES of the stabilityemission performance of staged gas-turbine combustion, $\mathrm{PhD}$ thesis, Institut National Polytechnique de Toulouse.

Schönfeld, T. and Rudgyard, M. (1999), 'Steady and Unsteady Flow Simulations Using the Hybrid Flow Solver AVBP', AIAA Journal 37(11), 1378-1385.

Shur, M., Strelets, M., Travin, A., Spalart, P. and Suzuki, T. (2018), 'Unsteady Simulations of a Fan/Outlet-GuideVane System: Aerodynamics and Turbulence', AIAA Journal 56(6), 2283-2297.

Wang, G., Duchaine, F., Papadogiannis, D., Duran, I., Moreau, S. and Gicquel, L. (2014), 'An overset grid method for large eddy simulation of turbomachinery stages', Journal of Computational Physics 274, 333-355.

Woodward, R., Hughes, C., Jeracki, R. and Miller, C. (2002), Fan Noise Source Diagnostic Test - Far-field Acoustic Results, in '8th AIAA/CEAS Aeroacoustics Conference \& Exhibit', AIAA-2002-2427, AIAA papers, Breckenridge, CO, USA. 